\title{
Does testing initiate decay of McCollough effects?
}

\author{
D. SKOWBO, S. GARRITY, and L. MICHAUD \\ Colby College, Waterville, Maine
}

\begin{abstract}
Results of several experiments (e.g., Jones \& Holding, 1975) have suggested that McCollough effects (MEs) decay little, if at all, in the absence of testing. We attempted to confirm these reports in a paradigm different from that used in previous work. The earlier findings did not generalize to our methodology: MEs measured some number of hours after induction were similar regardless of whether an initial test had been administered previously. We discuss possible explanations for the discrepancy between our results and previous findings. We speculate that a key methodological feature is the luminance of the test pattern.
\end{abstract}

McCollough effects (MEs), first reported 20 years ago (McCollough, 1965), are perceptual aftereffects of color whose appearance depends on the spatial configuration of lined patterns. For example, following inspection of red and black lines in a vertical orientation alternating with green and black lines oriented horizontally, black-andwhite grating patterns will appear tinted with weakly saturated hues approximately complementary to those paired with each orientation during the induction process. In this example, the white portion of vertical gratings would appear greenish and the white portion of horizontal patterns would appear pinkish.

One of the most striking features of MEs is their longevity. Unlike many other aftereffects, they commonly persist for several hours and may, under some circumstances, last for days or even weeks (see, e.g., Jones \& Holding, 1975). The normal time course of ME decay has been described by several investigators. Riggs, White, and Eimas (1974) found that MEs declined rapidly at first, then more slowly; the majority of their decay functions were nearly linear with log time after induction. MacKay and MacKay (1974) described ME decay with a negative power function over time since induction. An extensive discussion of ME decay appeared in Shute's (1979) monograph; his functions (which show ME decrement, rather than ME strength, on the ordinate) were linear on log$\log$ coordinates.

Deviations from normal ME decay may be effected in several ways. According the Shute (1979), various drugs may either accelerate or slow ME decay; nicotine, for example, has been reported to reduce rate of decay (see Amure, 1978). MacKay and MacKay $(1975,1977)$ found that MEs decay little during sleep or other periods of darkness lasting several hours. If the visual system is stimulated after ME induction, a prolonged exposure to achromatic patterns similar in spatial composition to the

The authors thank G. Cummings, C. Hyun, M. Hagerty, S. Martin, K. Melino, and E. Pendleton for serving as subjects. S. Garrity is now at New England Nuclear/DuPont, Billerica, MA. Requests for reprints should be sent to D. Skowbo, Department of Psychology, Colby College, Waterville, ME 04901. induction stimuli will cause MEs to decay unusually rapidly (Skowbo, Gentry, Timney, \& Morant, 1974).

Because assessment of the presence or magnitude of MEs entails having subjects view achromatic patterns, repeated testing might be expected to facilitate rapid decay. Jones and Holding (1975) designed an experiment to test this hypothesis. They compared ME decay in a group of subjects tested at five intervals $(0,8,24,56$, and $120 \mathrm{~h}$ after induction) with decay in five other groups, each tested for the first time at one of these intervals. MEs were assessed via a nulling technique-subjects rotated a color mixer, through which the test slide was projected, until the white portions appeared as colorless as possible. The median of three adjustments was taken as the index of ME strength. Although the repeated-test group showed a linear decrease over the geometric time intervals, the time-elapse groups showed little decay. Results of subsequent studies were not consistent with these findings, however. According to Favreau (1979) and White (1976/1977) MEs do not show more rapid decline with repeated testing. Shute (1979) reported that increasing the intertest interval by a factor of three was not associated with more rapid loss, but also expressed the opinion that very frequent or very infrequent testing could accelerate or slow decay.

The delayed-test groups in the Jones and Holding (1975) experiment were, indeed, tested very infrequently-not at all until the end of the delay period. When the first four delayed-test groups were retested at $120 \mathrm{~h}$, however, none of the retest means were significantly greater than zero. Because the MEs had decayed very little until the delayed initial test, but showed a substantial decline at the 120-h retest, Jones and Holding speculated that "a single prior test may be sufficient to begin the decay process" (p. 326). Jones and Holding reported additional data collected at even longer time-elapse test intervals, which ranged from about 10 days to nearly 3 months. MEs at approximately half strength or better were measured at all delay periods.

In a subsequent paper, Holding and Jones (1976) described an experiment designed to investigate the nature of delayed ME decay. In that study, initial time-elapse 
tests were made at 4,24 , or $96 \mathrm{~h}$ after induction. The assessment technique was the same as in the previous paper; subjects were exposed to the test slide for approximately $15-45 \mathrm{sec}$ in all. The results of tests made at the 24- and 96-h delays were significantly lower than the results of tests at 4 and $0 \mathrm{~h}$ (the latter data point was reproduced from the 1975 study), but the plotted means of all three time-delay tests fell well above the decay function of the repeated-test group from the 1975 experiment. Results of additional tests made after the delayed first assessment suggested that the first test initiated a decay process similar to that occurring with repeated testing.

Shute (1979) also reported some data on the effects of delayed initial tests. In one run, the ME was induced binocularly; one eye was tested immediately afterward, and the other, $36 \mathrm{~min}$ later. At that time, greater decrement was found in the eye that had been tested immediately. In another run, both eyes were tested, but the test was delayed until $1 \mathrm{~h}$ after induction. The decrement was described as about half what it normally would have been. Shute described the ability of MEs to remain relatively intact, beginning to decline substantially only upon the presentation of the test stimulus, as the most significant feature of MEs.

However, there is evidence in the literature that MEs do not always decay following initial testing: as noted above, work by MacKay and MacKay suggested that the effects do not decay in the absence of retinal stimulation. In their 1977 study, after induction and initial measurement of MEs, one eye was covered for $24 \mathrm{~h}$ while the other eye was tested periodically. Tests made upon removal of the eye patch indicated that the ME had not diminished in the preceding period; it then did begin to decay in a fashion very similar to that shown by the effect in the other eye the previous day. Evidently, then, delayed testing is not a necessary condition for prolonged ME storage, although it may be a sufficient one.

We agree with Shute (1979) that storage of the ME in the absence of testing would have strong theoretical significance. However, we believe that some of the data described above were not strong tests of the hypothesis that such storage exists. The data Shute reported were obtained in only two sessions with the same subject. In evaluating these data, particularly that from the second run, we note that day-to-day variability in MEs is often high (see, e.g., Skowbo \& Rich, 1982), and so a comparison between a single induction and an average or typical run may not be reliable.

The Jones and Holding (1975) experiment did not specifically test the hypothesis that a single test initiates decay; rather, this proposition was offered as a possible explanation for the results of the experiment. The focus of the Holding and Jones (1976) study was the decay that followed delayed tests. Although differences among the various group means did suggest an effect due to initial testing, no comparisons were possible between conditions equivalent except for the presence or absence of a single previous test.
We decided to test this hypothesis in a paradigm involving within-subjects comparisons between (1) tests administered for the first time after a delay and (2) tests administered for the second time after the same delay, a prior test having been made immediately after ME induction. If the Holding and Jones findings generalized to our paradigm, then a subject tested immediately after induction and then again at, say, $6 \mathrm{~h}$ would show a weaker ME than the same subject tested for the first time at $6 \mathrm{~h}$ after induction.

\section{EXPERIMENT 1}

\section{Method}

Subjects. Four females and four males participated. Results of the Farnsworth-Munsell 100-Hue Test indicated that each had colordiscriminating ability in the superior range. Before the experiment began, each was given practice in matching weakly saturated hues with the color-mixing device described below. Five of the subjects had never acquired MEs, two had seen an ME in a classroom demonstration several months before the experiment began, and one had been a participant in an ME experiment 2 years earlier.

Apparatus and Stimuli. The subjects sat facing a piece of acrylic rear-projection material mounted on a black background; induction and test patterns were projected onto this surface. All stimuli were circular, with a diameter of $21^{\circ}$.

Induction slides were constructed from 1.75-cycle/deg gratings and one of two Kodak Wratten filters, either No. 53 or No. 32. A neutral density filter (Wratten No. 96) was used in conjunction with No. 32 to make the luminances of the two induction stimuli more nearly equal. The space-averaged luminances of the green and magenta patterns were, respectively, 28 and $26 \mathrm{~cd} / \mathrm{m}^{2}$. An achromatic homogeneous stimulus used for light-adaptation purposes had a luminance of $1.5 \mathrm{~cd} / \mathrm{m}^{2}$.

The achromatic test stimulus consisted of a vertical grating on the top half and a horizontal grating on the bottom. In its center was an unlined circular area $7^{\circ}$ in diameter. A homogeneous field of variable chromaticity was projected onto this portion of the screen; subjects adjusted its appearance to match that of MEs visible on the surrounding gratings. The source of this field was a projection colorimeter modeled after a design by Riggs (1964). It mixed light transmitted by two Kodak Wratten color-compensating filters (No. CC30M and No. CC50G). The proportion contributed to a particular match by each filter is used to locate the mixture in C.I.E. space. In our particular system, all combinations made by these two filters lie along a straight line connecting two points with the coordinates $x=.407, y=.454$ and $x=.421, y=.350$. The luminance of the homogeneous central area was $1 \mathrm{~cd} / \mathrm{m}^{2}$, and the space-averaged luminance of the lined surround was $.6 \mathrm{~cd} / \mathrm{m}^{2}$ (lowluminance test patterns are associated with strongest assessed MEs; see White, 1976).

Procedure. Subjects began each session with $1 \mathrm{~min}$ of light adaptation, after which the test stimulus appeared. In order to determine whether residual effects from previous sessions were present, the subjects were asked to comment on the appearance of the test slide and to match, in ABBA sequence, the appearance of the vertical and horizontal lined portions. Next, the subjects viewed the induction stimuli, one oriented vertically and the other horizontally, alternating every $10 \mathrm{sec}$ for $10 \mathrm{~min}$. If the condition involved no immediate test, the subject left at that point. If the condition did require an immediate test, the subject viewed the light-adaptation slide for $1 \mathrm{~min}$ and subsequently matched the test stimulus once again as previously. The amount of time required to make these four matches varied somewhat from subject to subject; a typical duration of exposure to the test slide was approximately 1 to $1.5 \mathrm{~min}$. Delayed tests involved the same light-adaptation 


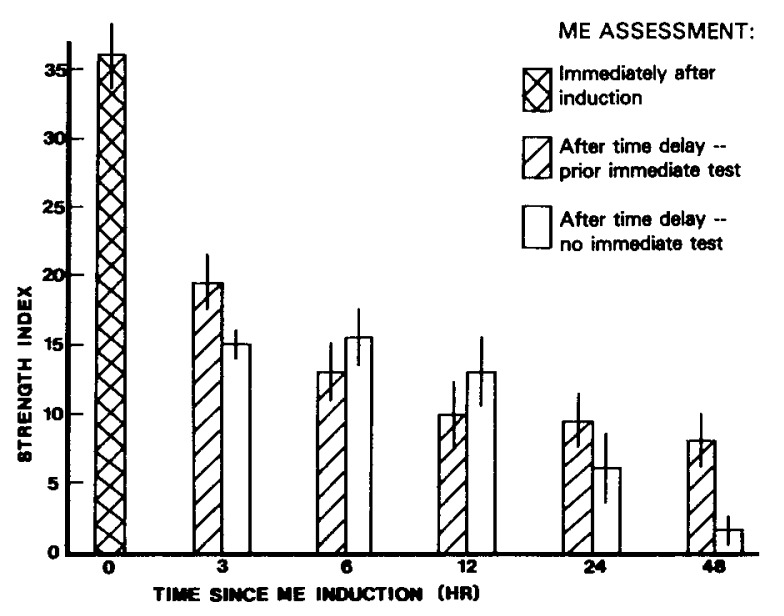

Figure 1. Assessed ME strength at various times following induction. Units on the ordinate are distances in C.I.E. space $\times 10^{3}$. The bars along the abscissa show ME strength immediately after induction and at the five time delays in conditions with and without an initial test. Data represented by the cross-hatched bar at time zero were obtained from the sessions in which there was an initial test; the height of this bar is a mean of $\mathbf{4 0}$ assessments, the 5 immediate tests for each of the 8 subjects. The heights of the other bars are means of 8 assessments, 1 for each subject. The vertical lines across the tops of the bars show \pm 1 estimated standard error from these means.

and matching procedure. They were scheduled at $3,6,12,24$, or $48 \mathrm{~h}$ after induction.

Each subject participated in 10 sessions, 2 for each time delay. Of these two, one condition involved a test immediately after induction plus a second test at the end of the time period. In the other condition, there was only one test, administered when the time period had elapsed.

The order of the conditions was essentially random, varying from subject to subject according to each individual's schedule. The color/orientation combination in the induction stimuli was reversed in successive sessions. At least $\mathbf{4}$ days elapsed between sessions.

\section{Results}

MEs were assessed by converting the mean of the two matches at each test orientation to C.I.E. coordinates; then a simple index of ME strength was taken as the distance in C.I.E. space between loci corresponding to the two means. Residual effects from session to session were not observed for the most part, but did appear to be present at the beginning of 3 of the 80 sessions. $^{1}$

Examination of the five immediate tests made by each subject indicated individual differences in initial strength of MEs. An ANOVA applied to these data did not yield a significant $F$ ratio, however, so data from all the subjects were combined in order to present the results in a condensed fashion.

Figure 1 shows the two test conditions at each timedelay period. Comparisons across time-delay periods show a fairly typical ME decay function, but there are no systematic differences within time periods associated with the presence versus absence of an initial test. Withinsubjects comparisons of ME strength in the two test conditions were made for all time periods. Out of $40 \mathrm{com}$ - parisons, there were 17 instances in which the delay-testonly condition produced a stronger assessed ME than was observed in the corresponding immediate-plus-delay condition and 23 instances in which the effect was weaker in the delay-test-only condition.

\section{EXPERIMENT 2}

Because subjects participated only once in each cell of the design of Experiment 1, we were concerned that session-to-session variability might have concealed a weak, but systematic, effect due to test schedule. We therefore decided to repeat a portion of Experiment 1 with multiple observations per subject in each cell. We reasoned, from results of previous studies, that, if an effect due to initial testing were present, it would be most likely to appear at one of the two extremes of the delay periods in Experiment 1: Shute's (1979) findings were obtained with delays of $1 \mathrm{~h}$ or less; the two experiments by Holding and Jones, on the other hand, revealed greatest effects at delays of $24 \mathrm{~h}$ or more.

\section{Method}

Subjects. Two females participated. One had served in Experiment 1 . The other, who had been a participant in numerous other ME studies, also had superior color-discriminating ability and was familiar with the color-matching task.

Apparatus and Stimuli. The apparatus was the same as in Experiment 1 , and the stimuli were constructed from the same materials. However, a routine replacement of projector bulbs and gelatin filters took place between Experiments 1 and 2, resulting in somewhat different luminance values for stimuli as follows (all values for gratings are space-averaged): light-adaptation stimulus, $1.2 \mathrm{~cd} / \mathrm{m}^{2}$; test grating, $.5 \mathrm{~cd} / \mathrm{m}^{2}$; green induction grating, $23 \mathrm{~cd} / \mathrm{m}^{2}$;

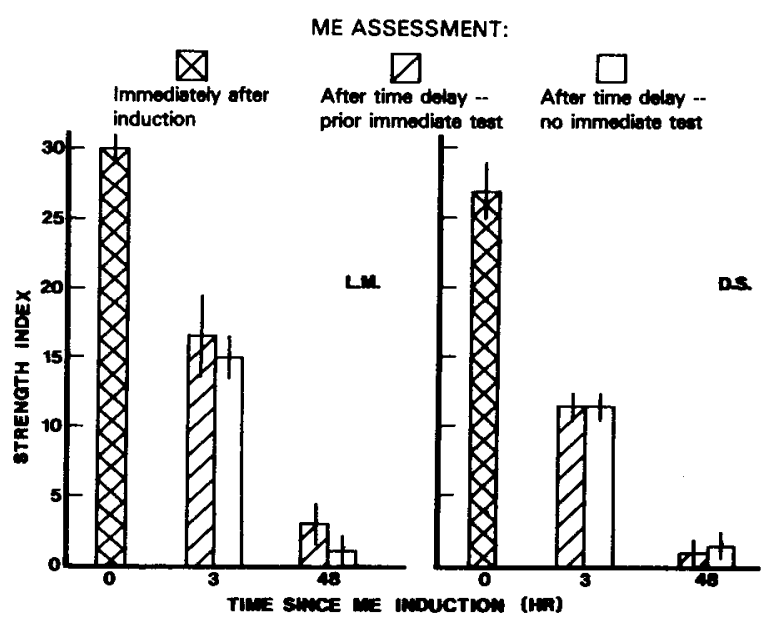

Figure 2. Assessed ME strength for two subjects at 0,3 , and 48 h after induction. Units on the ordinate are the same as thoee described in Figure 1. Bars along the abscissa at 3 and $48 \mathrm{~h}$ show ME strength in the conditions with and without an initial test. The heights of these bars are means of three sessions, and the heights of the croes-hatched bars at time zero are means of six assessments obtained in the runs containing an initial test. Vertical lines across the top of each bar show \pm 1 estimated standard error from these means. 
magenta induction grating, $21 \mathrm{~cd} / \mathrm{m}^{2}$. The luminance of the matching spot did not change.

Procedure. The procedure was the same as in Experiment 1. However, only the 3-and the 48-h time periods were used. Each subject participated three times in the two conditions at each time period, or 12 times in all.

\section{Results}

The strength index for MEs was calculated in the same manner as in Experiment 1. No residual effects from previous sessions were ever observed.

Figure 2 shows the means of the three ME assessments for the two test conditions at the two delay periods. Data from the two subjects were not significantly different, but their assessments are shown separately in order to present these results in an uncondensed fashion. Again, as in Experiment $1, \mathrm{ME}$ measures at time delays were not stronger when those measures were the first, rather than the second, assessments.

\section{DISCUSSION}

Results of our two experiments are not consistent with the results of Holding and Jones (1976), Jones and Holding (1975), and Shute (1979). As noted earlier, Shute presented data from only two sessions, and no methodological details were given. Therefore, we limit our concern in this section to discrepancies between our results and those from the experiments of Holding and Jones.

A major methodological difference between the two paradigms is that we used repeated measures and they did not. In the Holding and Jones experiments, all subjects were previously naive to MEs and acquired an ME only once; testing then took place on one or several occasions following that single induction. In our experiments, however, the ME was induced in each subject a number of times. Holding (personal communication, September 26,1984 ) suggested that our within-subjects comparisons might be confounded by prior treatments. In our opinion, there is little, if any, evidence that MEs show longterm carryover effects (see, e.g., Skowbo \& Rich, 1982); but even if such effects did exist, it is unclear how previous exposure to MEs would prevent them from persisting in the absence of initial testing. The history of the subject would have to interact with the test schedule such that only naive subjects would retain MEs if an initial test was not administered. Despite our reservations about this possibility, we attempted to evaluate it by examining data from the five subjects with no exposure to MEs prior to the first session of Experiment 1-by chance, the first session for all five had been a delay-test-only condition. We compared scores from these first sessions to the means of their various time-delay groups. In one case, the firstsession assessment was at the group mean, in two it was greater than the mean, and in two it was below the mean.

We considered a related possibility that naive subjects might show unusually long persistence of MEs regardless of the testing schedule. In a brief supplementary experiment, four naive subjects acquired MEs and were tested
5-6 $\mathrm{h}$ later on three successive occasions; for two of these four subjects, an initial test immediately after induction was also administered. However, there was no tendency for the first session to contain the strongest of the three time-delay MEs.

Further comparisons of the two paradigms reveal differences in several parameters known to affect the assessed strengths of MEs. For example, relative to their stimuli, our inspection patterns were higher in luminance and in spatial frequency. These conditions would be expected to induce stronger MEs in our subjects (see Stromeyer, 1972; White, 1976), a tendency that would be offset somewhat by Holding and Jones's longer inspection duration (see Riggs et al., 1974). The rate at which MEs decay depends on their initial strength (see Riggs et al., 1974; Shute, 1979), and the net effect of these conditions might be a tendency for subjects in one paradigm to show a faster rate of decay, overall, than subjects in the other. By themselves, though, these different conditions do not explain why the presence versus absence of a prior test seemed to affect MEs within one paradigm but not within the other.

Holding and Jones also used relatively intense test patterns. The luminance reported for their achromatic stimulus was not only higher than that of our test stimulus, but even slightly higher than that given for their induction stimuli. High-luminance test patterns are associated with low assessed MEs (White, 1976). Also, postinduction exposure to high-luminance achromatic patterns has been shown to weaken MEs more than exposure to lowluminance patterns (Skowbo, 1979). Thus, this test condition would have favored the hypothesis that repeated assessments could interfere with MEs. It might also help to explain why Holding and Jones observed substantial declines after even a single test.

In the Jones and Holding (1975) paper, the proposition that testing initiated decay was inspired by discrepancies between several groups tested for the second time at $120 \mathrm{~h}$ and the group tested for the first time at $120 \mathrm{~h}$. Comparisons among the various time-elapse conditions of the 1976 study also suggested that decay began after a single test. For example, subjects tested for the first time after 24 and $96 \mathrm{~h}$ had slightly stronger effects than subjects tested for only the second time at $8 \mathrm{~h}$ after induction. We do not know whether the effects of a fairly brief exposure to their test grating would be sufficiently deleterious to account for differences such as these on the basis of luminance of the test pattern rather than testing per se. However, White (1976) has stressed that test pattern and inspection pattern luminances interact in their effects on ME assessments made over time since induction. We used a combination of high-luminance inspection patterns and low-luminance test patterns, whereas Holding and Jones used inspection and test patterns fairly similar in luminance, with values intermediate between our two extremes. Perhaps their particular stimulus conditions foster the generation of slowly decaying effects that are markedly subdued by testing, whereas ours favor relatively rapidly 
decaying effects which are not further depressed by testing. Further research is needed to clarify the interactions among these parameters.

In sum, we have found that MEs decay similar amounts regardless of whether or not they are tested immediately after induction. When low-luminance test patterns are used, testing does not appear to initiate ME decay. If MEs can be retained prior to testing, then they do so only within methodological constraints as yet to be specified.

\section{REFERENCES}

AMURE, B. (1978). Nicotine and the decay of the McCollough effect. Vision Research, 18, 1449-1451.

FAVREAU, O. (1979). Persistence of simple and contingent motion aftereffects. Perception \& Psychophysics, 26, 187-194.

Holding, D., \& JoNES, P. (1976). Delayed one-trail extinction of the McCollough effect. Quarterly Journal of Experimental Psychology, 28, 683-687.

JoNEs, P., \& HoLDING, D. (1975). Extremely long-term persistence of the McCollough effect. Joumal of Experimental Psychology: Human Perception and Performance, 1, 323-327.

MacKAY, D., MACKAY, V. (1974). The time course of the McCollough effect and its physiological implications. Journal of Physiology (London), 327, 38.

MACKAY, D., \& MACKAY, V. (1975). What causes decay of patterncontingent chromatic aftereffects? Vision Research, 15, 462-464.

MacKaY, D., MacKaY, V. (1977). Retention of the McCollough effect in darkness: Storage or enhanced read-out? Vision Research, 17, 313-315.

McCollough, C. (1965). Color adaptation of edge detectors in the human visual system. Science, 149, 1115-1116.

RIGGS, L. (1964). A projection color mixer. American Journal of Psychology, 77, 129-134.

RigGS, L., WhIte, K., \& Eimas, P. (1974). Establishment and decay of orientation-contingent aftereffects of color. Perception \& Psychophysics, 16, 535-542.
SHUTE, C. (1979). The McCollough effect: An indicator of central neurotransmitter activity. Cambridge: Cambridge University Press.

Sxowbo, D. (1979). Luminance as a factor in the ability of achromatic gratings to interfere with McCollough effects. Perception \& Psychophysics, 26, 105-107.

Skowbo, D., Gentry, T., Timney, B., \& Morant, R. (1974). The McCollough effect: Influence of several kinds of visual stimulation on decay rate. Perception \& Psychophysics, 16, 47-49.

Skowbo, D., \& Rich, J. (1982). Practice does not facilitate acquisition of McCollough effects: Evidence against a learning model. Perception \& Psychophysics, 32, 551-554.

STROMEYER, C. (1972). Edge-contingent color aftereffects: Spatial frequency specificity. Vision Research, 12, 717-732.

WHITE, K. (1976). Luminance as a parameter in establishment and testing of the McCollough effect. Vision Research, 16, 297-302.

WhITE, K. (1977). Studies of orientation-contingent aftereffects (Doctoral dissertation, Brown University, 1976). Dissertation Abstracts International, 38, 403. (University Microfilms No. AAD77-14207)

\section{NOTES}

1. All three of these occasions involved the same male subject, who appeared at these times to have slight residual MEs from sessions held 1 week or more earlier. On one of these occasions, however, the association between color and orientation was the opposite of that in the previous ME. Because time constraints imposed by his impending graduation made longer delays between sessions impossible, he was run on these occasions nevertheless. Data from these three sessions did not appear unusual in any way, nor were data from this subject systematically different from those of other subjects. Also, a version of Figure 1 prepared with this subject's data excluded did not show a different pattern among the various means. Therefore, these data are included in the results.

(Manuscript received November 13, 1984; revision accepted for publication March 28, 1985.) 